\title{
Role of Poly Adenosine Diphosphate Ribose Polymerase Inhibitors in Advanced Stage Ovarian Cancer
}

\author{
Ena Arora ${ }^{1}$, Muhammad Masab ${ }^{2}$, Vishal Jindal ${ }^{3}$, Iqra Riaz ${ }^{4}$, Sorab Gupta ${ }^{5}$, Gabor Varadi ${ }^{5}$ \\ 1. Obstetrics and Gynecology, Civil Hospital Chandigarh, Chandigarh, IND 2. Internal Medicine, Albert Einstein \\ Medical Center, New York, USA 3. Internal Medicine, St. Vincent Hospital Worcester, Worcester, USA 4. Department of \\ Medicine, Mayo Hospital, King Edward Medical University, Lahore, Pakistan 5. Hematology and Oncology, Albert \\ Einstein Medical Center, New York, USA
}

Corresponding author: Muhammad Masab, masabmuh@einstein.edu

\begin{abstract}
Ovarian cancer is one of the leading causes of death from gynecologic cancers. In this present era of cancer treatment, therapeutic options for patients with advanced or recurrent ovarian cancer are limited. The present standard of care treatment for advanced ovarian cancer is a platinum-based doublet chemotherapy (paclitaxel and carboplatin with or without bevacizumab) after a maximum attempt of surgical cytoreduction. However, there are no promising options for the management of patients with ovarian cancer refractory to the platinum-based chemotherapy. Therefore, newer, safe, and more effective treatment modalities are required for patients with advanced or recurrent ovarian cancer. Poly(adenosine diphosphate [ADP]-ribose) polymerase (PARP) inhibitors have shown an impressive safety profile and anti-tumor efficacy in patients with breast cancer 1 and 2 (BRCA1 and BRCA2) gene-mutated ovarian cancer who were previously treated with the standard of care chemotherapy. We have done a detailed review of the literature to emphasize the role of PARP inhibitors in the treatment of advanced or relapsed ovarian cancer.
\end{abstract}

Received 05/01/2018

Review began 05/13/2018 Review ended 05/17/2018 Published 05/24/2018

(c) Copyright 2018 Arora et al. This is an open access article distributed under the terms of the Creative Commons Attribution License CC-BY 3.0., which permits unrestricted use, distribution, and reproduction in any medium, provided the original author and source are credited.
Categories: Internal Medicine, Obstetrics/Gynecology, Oncology

Keywords: ovarian cancer, parp inhibitors, olaparib, rucaparib, niraparib, poly (adenosine diphosphate [adp]-ribose) polymerase (parp)

\section{Introduction And Background}

Ovarian cancer is one of the leading causes of death from gynecologic cancers. It is the fifth most common cause of cancer-related deaths amongst women of the United States. In 2018, epidemiological studies have shown that there will be around 22,240 new diagnoses and 14,070 deaths from ovarian cancer in the United States. The five-year survival rate from ovarian cancer is up to $46.5 \%$. Also, the cure rate is around $40 \%$ [1-2]. The median age of diagnosis is 63 years, and the majority of women (70\%) have advanced disease at presentation [1-2].

Some of the main risk factors associated with ovarian cancers are late pregnancy, nulliparous women, hormone replacement therapy, pelvic inflammatory disease, endometriosis, and genetic mutations [3-5]. Patients having two or more first-degree relatives with ovarian cancer usually have an early onset of the disease, especially with the breast cancer gene 1 and 2 (BRCA1 and BRCA2) germline mutations or Lynch syndrome [6-18].

Human cells continue to develop mutations throughout their life that can lead to cell death if the damage is extensive. Moreover, sometimes mutations can also allow a cell to acquire a malignant potential. There are different deoxyribonucleic acid (DNA) repair mechanisms to neutralize these mutations. Some of these repair pathways are nucleotide-excision, base-excision, homologous recombination, mismatch repair, and telomerase metabolism.

Poly(adenosine diphosphate [ADP]-ribose) polymerases (PARPs) are a group of enzymes that carry on the modification of post-translational proteins. PARP-1 and PARP-2 are the main subtypes of the PARP family, that are implicated in the base-excision repair (BER) of single-stranded breaks (SSBs) [19-20]. PARPs also play an important role in the homologous recombination (HR) pathway. It identifies the disrupted replication forks and works with the DNA protein kinase complex involved in non-homologous end-joining (NHEJ) [21]. BRCA1 and BRCA2 have a significant role in the double-stranded repair through the HR pathway [22]. Cells with mutations in BRCA1 or BRCA2 lose the function to repair double-stranded breaks and thus, utilize NHEJ for DNA repair. PARP inhibition is not deadly to cells without the BRCA mutation because of a normal working HR pathway. As cells with a BRCA mutation are deficient in the HR pathway, the loss of PARP activity in such cells will result in cell death. Synthetic lethality was the term coined for this mechanism, and it led to further research of the PAPR inhibition [23-24]. 
PARP inhibitors work by attaching to the nicotinamide adenine dinucleotide (NAD+) binding site of the PARP molecule, thereby inhibiting its function. Various studies have shown that BRCA-mutated cells are significantly affected by PARP inhibition [25-26].

\section{Review}

\section{Olaparib}

Olaparib is a PARP inhibitor approved for the management of ovarian cancer, both for initial as well as maintenance treatment. It showed impactful response rates in the BRCA1 or BRCA2-mutated platinumsensitive ovarian cancer patients [27-32].

SOLO2/ENGOT-OV21A was a clinical trial evaluating olaparib as a maintenance treatment in BRCA 1/2 platinum-sensitive, relapsed ovarian cancer patients who had received two or more lines of chemotherapy (registered with ClinicalTrials.gov, \#NCT01874353). It included patients with high-grade serous, endometrial, primary peritoneal, or fallopian tube cancer. Two hundred and ninety-five eligible patients were randomly assigned to receive olaparib or a placebo treatment. This trial showed a longer median progression-free survival with olaparib, which was significant (19.1 months compared with 5.5 months for placebo). In the olaparib group, 35 (18\%) patients showed serious adverse events as compared to the placebo group (8\%). The common adverse events of Grade 3 or worse severity were anemia (19\% in the olaparib group vs $2 \%$ in the placebo group), fatigue (4\% in olaparib group vs $2 \%$ in placebo group), and neutropenia (5\% in olaparib group vs $4 \%$ in placebo group) [33].

Another randomized, double-blinded, placebo-controlled, Phase 2 study (registered with ClinicalTrials.gov, \#NCT00494442) evaluated olaparib in the maintenance settings in patients with platinum-sensitive, relapsed, high-grade serous ovarian cancer who had received two or more platinum-based regimens with a partial or complete response [32]. Two hundred and sixty-five patients were enrolled, and regardless of BRCA status, they were randomly assigned to receive $400 \mathrm{mg}$ twice daily of olaparib vs placebo. This study showed a statistically significant improvement in progression-free survival (PFS) in patients treated with olaparib (8.4 months) as compared to placebo (4.8 months). The adverse events observed were mainly Grade 1 or 2 in the olaparib group and these were nausea (68\%), fatigue (49\%), vomiting (32\%), and anemia (17\%).

Based on these clinical trials, the Food and Drug Administration (FDA) and the National Comprehensive Cancer Network (NCCN) guidelines recommended olaparib to be used as a maintenance therapy for women with advanced stage ovarian cancer who have received two or more lines of chemotherapy.

The FDA also approved olaparib as a single agent for the treatment of advanced ovarian cancer patients with germline BRCA1 or BRCA2 mutation who had received three or more prior lines of chemotherapy. This approval was based on the results of a single arm Phase 2 study, that enrolled 298 patients with solid tumors and BRCA1 or BRCA2 mutations [34]. All patients had received prior platinum-containing chemotherapy and were considered to be platinum-resistant or not suitable for further platinum therapy. Of the 193 patients in the ovarian cancer cohort, 178 had ovarian cancer, four had fallopian tube cancer, and 11 had primary peritoneal cancer. All patients were pretreated with chemotherapy with a mean number of prior regimens up to 4.3. Response rates were $31.1 \%$ in patients with ovarian cancer. Patients had a median PFS of seven months, a median overall survival (OS) of 16.6 months, and one year OS of $64.4 \%$ [34]

\section{Rucaparib}

Rucaparib is an oral PARP inhibitor used in advanced (Stage III-IV) ovarian cancer. A Phase 2 trial (ARIEL-2) (registered with ClinicalTrials.gov, \#NCT01891344) evaluated the safety and efficacy of rucaparib in the treatment of relapsed platinum-sensitive ovarian cancers in patients who had received at least two prior lines of platinum-based chemotherapy. A total of 192 patients were divided into three subgroups: BRCA mutant, BRCA wild-type with high loss of heterogeneity (LOH) defined as $14 \%$ or more genomic LOH (LOH high group), or BRCA wild-type with less than 14\% genomic LOH (LOH low group). Rucaparib was given continuously at a dose of $600 \mathrm{mg}$ twice per day (28-day cycle) until the patients had disease progression or developed an adverse event. The primary endpoint was median progression-free survival (PFS). The median PFS was 12.8 months in the BRCA mutant group, 5.7 months in the LOH high group, and 5.2 months in the LOH low group. Patients in the LOH high group had improved responses to rucaparib (39\% compared to 69\% for the BRCA mutation subgroup and 11\% for the BRCA wild-type, LOH low patients) [9]. Common Grade 3 or more adverse events observed with the drug were anemia (22\%) and elevations in alanine aminotransferase or aspartate aminotransferase (12\%) [35].

The results of this trial showed the efficacy of rucaparib in BRCA-mutant patients and also suggested that the assessment of genomic $\mathrm{LOH}$ can help to identify platinum-sensitive ovarian cancer patients with BRCA wild-type who can respond to rucaparib.

\section{Niraparib}

Niraparib is an oral PARP inhibitor which was investigated in a randomized, double-blind, Phase 3 trial 
(NOVA) to study the clinical efficacy of maintenance niraparib in platinum-sensitive, recurrent ovarian cancer patients who had received at least two previous lines of platinum-based treatment (registered with ClinicalTrials.gov, \#NCT01847274) [36]. This study enrolled 597 patients with a BRCA1/2 germline mutation, as well as the BRCA wild-type. BRCA wild-type patients were further stratified on the basis of homologous recombination deficiency (HRD). Patients were randomly allocated to receive niraparib (300 mg) or placebo once daily. The primary endpoint was PFS.

Patients in the niraparib group had a significantly longer median PFS as compared to the placebo group (21.0 months vs 5.5 months, respectively) in the BRCA-mutant group. The median PFS was also improved in the BRCA wild-type group with positive HRD who received niraparib compared to placebo (12.9 months vs. 3.8 months, respectively). The median PFS for niraparib in patients with overall BRCA wild-type group, irrespective of HRD, was 9.3 months vs. 3.9 months for the placebo. The most common Grade 3 or 4 adverse events in the niraparib group were thrombocytopenia (33.8\%), anemia (25.3\%), and neutropenia (19.6\%) [36].

The NOVA trial results demonstrated that median PFS for patients with platinum-sensitive, recurrent ovarian cancer was significantly longer in patients who received niraparib as compared to placebo, irrespective of the BRCA mutation or HRD status [36].

\section{Conclusions}

In summary, PARP inhibitors demonstrate a very promising safety profile and anti-tumor efficacy in patients with BRCA1 or BRCA2-mutated platinum-sensitive ovarian cancer patients in both treatment and maintenance settings. The recent clinical studies are also encouraging to illustrate its role in some platinum refractory and BRCA wild-type ovarian cancer patients. More studies are needed for a better understanding of the use of PARP inhibitors in ovarian cancer.

\section{Additional Information}

\section{Disclosures}

Conflicts of interest: In compliance with the ICMJE uniform disclosure form, all authors declare the following: Payment/services info: All authors have declared that no financial support was received from any organization for the submitted work. Financial relationships: All authors have declared that they have no financial relationships at present or within the previous three years with any organizations that might have an interest in the submitted work. Other relationships: All authors have declared that there are no other relationships or activities that could appear to have influenced the submitted work.

\section{References}

1. Siegel RL, Miller KD, Jemal A: Cancer statistics, 2018. CA Cancer J Clin. 2018, 68:7-30. 10.3322/caac.21442

2. SEER Cancer Statistics Review (CSR) 1975-2014 . (2017). Accessed: April 30, 2018: http://seer.cancer.gov/archive/csr/1975_2014/.

3. Mørch LS, Løkkegaard E, Andreasen AH, et al.: Hormone therapy and ovarian cancer . JAMA. 2009, 302:298305. 10.1001/jama.2009.1052

4. Mørch LS, Løkkegaard E, Andreasen AH, et al.: Hormone therapy and different ovarian cancers: a national cohort study. Am J Epidemiol. 2012, 175:1234-42. 10.1093/aje/kwr446

5. Lin HW, Tu YY, Lin SY, et al.: Risk of ovarian cancer in women with pelvic inflammatory disease: a population-based study. Lancet Oncol. 2011, 12:900-904. 10.1016/S1470-2045(11)70165-6

6. Nakonechny QB, Gilks CB: Ovarian cancer in hereditary cancer susceptibility syndromes . Surg Pathol Clin. 2016, 9:189-99. 10.1016/j.path.2016.01.003

7. Chui MH, Ryan P, Radigan J, et al.: The histomorphology of Lynch syndrome-associated ovarian carcinomas: toward a subtype-specific screening strategy. Am J Surg Pathol. 2014, 38:1173-81. 10.1097/PAS.0000000000000298

8. Lu KH, Daniels M: Endometrial and ovarian cancer in women with Lynch syndrome: update in screening and prevention. Fam Cancer. 2013, 12:273-77. 10.1007/s10689-013-9664-5

9. Lancaster JM, Powell CB, Chen LM, et al.: Society of Gynecologic Oncology statement on risk assessment for inherited gynecologic cancer predispositions. Gynecol Oncol. 2015, 136:3-7. 10.1016/j.ygyno.2014.09.009

10. Rebbeck TR, Mitra N, Wan F, et al.: Association of type and location of BRCA1 and BRCA2 mutations with risk of breast and ovarian cancer. JAMA. 2015, 313:1347-61. 10.1001/jama.2014.5985

11. Daly MB, Axilbund JE, Buys S, et al.: Genetic/familial high-risk assessment: breast and ovarian. J Natl Compr Canc Netw. 2010, 8:562-94. 10.6004/inccn.2010.0043

12. Walsh CS, Blum A, Walts A, et al.: Lynch syndrome among gynecologic oncology patients meeting Bethesda guidelines for screening. Gynecol Oncol. 2010, 116:516-21. 10.1016/i.ygyno.2009.11.021

13. Lancaster JM, Powell CB, Kauff ND, et al.: Society of Gynecologic Oncologists Education Committee statement on risk assessment for inherited gynecologic cancer predispositions. Gynecol Oncol. 2007, 107:159-62. 10.1016/j.ygyno.2007.09.031

14. Shulman LP: Hereditary breast and ovarian cancer (HBOC): clinical features and counseling for BRCA1 and BRCA2, Lynch syndrome, Cowden syndrome, and Li-Fraumeni syndrome. Obstet Gynecol Clin North Am. 2010, 37:109-33. 10.1016/j.ogc.2010.03.003

15. ACOG Committee on Practice Bulletins: Hereditary breast and ovarian cancer syndrome. Gynecol Oncol. 2009, 113:6-11. 10.1016/j.ygyno.2009.02.017 
16. American College of Obstetricians and Gynecologists; ACOG Committee on Practice Bulletins--Gynecology; ACOG Committee on Genetics; Society of Gynecologic Oncologists: ACOG Practice Bulletin No. 103: Hereditary breast and ovarian cancer syndrome. Obstet Gynecol. 2009, 113:957-66.

10.1097/AOG.0b013e3181a106d4

17. Zhang S, Royer R, Li S, et al.: Frequencies of BRCA1 and BRCA2 mutations among 1,342 unselected patients with invasive ovarian cancer. Gynecol Oncol. 2011, 121:353-57. 10.1016/j.ygyno.2011.01.020

18. Liu G, Yang D, Sun Y, et al.: Differing clinical impact of BRCA1 and BRCA2 mutations in serous ovarian cancer. Pharmacogenomics. 2012, 13:1523-35. 10.2217/pgs.12.137

19. Houtgraaf JH, Versmissen J, van der Giessen WJ: A concise review of DNA damage checkpoints and repair in mammalian cells. Cardiovasc Revasc Med. 2006, 7:165-72. 10.1016/j.carrev.2006.02.002

20. El-Khamisy SF, Masutani M, Suzuki H, Caldecott KW: A requirement for PARP-1 for the assembly or stability of XRCC1 nuclear foci at sites of oxidative DNA damage. Nucleic Acids Res. 2003, 31:5526-33. 10.1093/nar/gkg761

21. Wang M, Wu W, Wu W, et al.: PARP-1 and Ku compete for repair of DNA double strand breaks by distinct NHEJ pathways. Nucleic Acids Res. 2006, 34:6170-82. 10.1093/nar/gkl840

22. Tutt A, Ashworth A: The relationship between the roles of BRCA genes in DNA repair and cancer predisposition. Trends Mol Med. 2002, 8:571-76. 10.1016/S1471-4914(02)02434-6

23. Bryant HE, Schultz N, Thomas HD, et al.: Specific killing of BRCA-2 deficient tumours with inhibitors of poly(ADP-ribose) polymerase. Nature. 2005, 434:913-17. 10.1038/nature03443

24. Farmer H, McCabe N, Lord CJ, et al.: Targeting the DNA repair defect in BRCA mutant cells as a therapeutic strategy. Nature. 2005, 917-921. 10.1038/nature03445

25. Langelier MF, Zandarashvili L, Aguiar PM, et al.: NAD+ analog reveals PARP-1 substrate-blocking mechanism and allosteric communication from catalytic center to DNA-binding domains. Nat Commun. 2018, 9:844. 10.1038/s41467-018-03234-8

26. Evers B, Drost R, Schut E, et al.: Selective inhibition of BRCA2-deficient mammary tumor cell growth by AZD2281 and cisplatin. Clin Cancer Res. 2008, 14:3916-25. 10.1158/1078-0432.CCR-07-4953

27. Elit L, Hirte H: Palliative systemic therapy for women with recurrent epithelial ovarian cancer: current options. Onco Targets Ther. 2013, 6:107-18. 10.2147/OTT.S30238

28. Suh DH, Lee KH, Kim K, et al.: Major clinical research advances in gynecologic cancer in 2014 . J Gynecol Oncol. 2015, 26:156-67. 10.3802/jgo.2015.26.2.156

29. Gelmon KA, Tischkowitz M, Mackay H, et al.: Olaparib in patients with recurrent high-grade serous or poorly differentiated ovarian carcinoma or triple-negative breast cancer: a phase 2, multicentre, open-label, nonrandomised study. Lancet Oncol. 2011, 12:852-61. 10.1016/S1470-2045(11)70214-5

30. Audeh MW, Carmichael J, Penson RT, et al.: Oral poly(ADP-ribose) polymerase inhibitor olaparib in patients with BRCA1 or BRCA2 mutations and recurrent ovarian cancer: a proof-of-concept trial. Lancet. 2010, 376:245-51. 10.1016/S0140-6736(10)60893-8

31. Fong PC, Yap TA, Boss DS, et al.: Poly(ADP)-ribose polymerase inhibition: frequent durable responses in BRCA carrier ovarian cancer correlating with platinum-free interval. J Clin Oncol. 2010, 28:2512-19. Accessed: May 24, 2018: 10.1200/JCO.2009.26.9589

32. Ledermann J, Harter P, Gourley C, et al.: Olaparib maintenance therapy in platinum-sensitive relapsed ovarian cancer. N Engl J Med. 2012, 366:1382-92. 10.1056/NEJMoa1105535

33. Pujade-Lauraine E, Ledermann JA, Selle F, et al.: Olaparib tablets as maintenance therapy in patients with platinum-sensitive, relapsed ovarian cancer and a BRCA1/2 mutation (SOLO2/ENGOT-Ov21): a doubleblind, randomised, placebo-controlled, phase 3 trial. Lancet Oncol. 2017, 18:1274-84. 10.1016/S14702045(17)30469-2

34. Kaufman B, Shapira-Frommer R, Schmutzler RK, et al.: Olaparib monotherapy in patients with advanced cancer and a germline BRCA1/2 mutation. J Clin Oncol. 2015, 33:244-50. 10.1200/JCO.2014.56.2728

35. Swisher EM, Lin KK, Oza AM, et al.: Rucaparib in relapsed, platinum-sensitive high-grade ovarian carcinoma (ARIEL2 Part 1): an international, multicentre, open-label, phase 2 trial. Lancet Oncol. 2017, 18:75-87. 10.1016/S1470-2045(16)30559-9

36. Mirza MR, Monk BJ, Herrstedt J, et al.: Niraparib maintenance therapy in platinum-sensitive, recurrent ovarian cancer. N Engl J Med. 2016, 375:2154-64. 10.1056/NEJMoa1611310 